\title{
Simulation Analysis of Wireless Channel Effect on IEEE 802.11n Physical Layer
}

\author{
Ali Bouhlel", Valéry Guillet* \\ *Engineering and Propagation Department \\ Orange Labs 6 av. des usines \\ 90007 Belfort Cedex, France \\ \{ali.bouhlel, valery.guillet\}@orange.com
}

\author{
Ghais El Zein ${ }^{+}$, Gheorghe Zaharia ${ }^{+}$ \\ ${ }^{+}$IETR - INSA, \\ UMR 6164, 20 av. des buttes de Coësmes, \\ CS 70839,35708 Rennes Cedex 7 \\ \{ghais.el-zein, gheorghe.zaharia\}@insa-rennes.fr
}

\begin{abstract}
IEEE 802.11n standard came as a rescue; the existing standards are increasingly seen as inadequate since applications become more complex and require more bandwidth. Several techniques have been put into operation to meet two basic requirements: significantly greater bit rate and radio coverage. However, studies have shown that the theoretical limit in terms of throughput is far from being reached and that the received power does not explain the performance degradation. A list of suspect parameters is analyzed in this paper to assess their effect on performance of the IEEE 802.11n physical layer taken as an application of MIMO technology in indoor context. It is shown that for values of angular spread below $27^{\circ}$, the data rate cannot exceed $117 \mathrm{Mbps}$ and the antennas spacing can compensate the performance degradation caused by other parameters. Results are given in terms of correlation coefficient, other channel characteristics and the packet error rate.
\end{abstract}

Keywords: MIMO, IEEE802.11n, WLAN, Packet Error Rate (PER), Channel models.

\section{INTRODUCTION}

The originality of the standard IEEE802.11n is based on the use of spatial multiplexing, facilitating the propagation and on an associated system of Multiple Input, Multiple Output antennas (MIMO). Other important enhancements concern transmission channels operating with $40 \mathrm{MHz}$ bandwidth and frame aggregation mechanisms to have a better MAC efficiency. This transmission system, which can reach in principle a throughput of 100 to $300 \mathrm{Mbps}$, is likely to extend a wired Ethernet protocol over a WLAN to provide interactive multimedia services to mobile users.

Because of the large number of parameters in question, system performance is variable, though the promises have been great. In all WLAN, the major difficulties come from the availability of frequencies, the nature of the propagation environment (indoor, outdoor, building materials...), and the used equipment. New difficulties are met in 802.11n networks, due to the large number of parameters combinations that can arise when, for each link, two transmitters and two receivers operating in parallel within the same MIMO channel and the number of data streams transmitted at each time increases. The term "MxN" is used to describe the number of antennas at each end of the transmission channel 802.11n. The minimum required by the standard is the so-called " $2 \times 2$ " (two transmitting antennas and two receiving antennas). Previous studies have proved the impact of the channel correlation properties [1].

Therefore, in Section II, a description of the wireless channel model is given and an identification of the different parameters involved in the quality of an IEEE 802.11n link is done. Then, an analysis of the effect of the various elements related to the propagation on the performance in terms of bit rate is presented in Section III. Section IV presents the impact of a couple of parameters on the PER assessed in order to identify relevant areas corresponding to a low packet error rate. The conclusion is drawn in Section V.

\section{SIMULATIONS DESCRIPTIONS AND CHANNEL MODEL}

\section{A. Simulations set up}

Matlab and Simulink were used in order to simulate $11 \mathrm{n}$ links. The transmission chain implements the new technologies of the IEEE 802.11 n physical layer [2]. The most important functionalities of the simulation block are:

- A 2x2 MIMO system

- A set of Modulation and Coding Schemes (MCS): only the $\mathrm{MCS}_{8 \ldots 15}$ could be used for two spatial streams. The BPSK, QPSK, QAM-16, QAM-64 modulations are used with the coding rates $(1 / 2,3 / 4,2 / 3,5 / 6)$

- OFDM (only $20 \mathrm{MHz}$ supported): 52 sub-carriers (data), 64 FFT points, with use of cyclic prefix

- MIMO Detection: a MMSE linear detector

- Antenna spacing at Tx and Rx array is $\lambda / 2$, where $\lambda$ is the wavelength

- Omni V polarized antennas 
- Support for TGn channel models which will be described in the next paragraph.

\section{B. Description of the channel model}

The propagation channel model used for the simulations is designed by the "High Throughput Task Group" (TGn). The Matlab script is described in [2]. Accurate explanations of the channel modeling can be found in [3] and [4].

The propagation channel imposes delay and phase shifts on the signals exchanged between the transmitter and the receiver. The received signals arrive in clusters, and each one is composed of several rays. The standard defines several channel models (A to $\mathrm{F}$ ) in order to model multiple propagation environments.

The use of A-F models has been presented in [5]. These models are representative for small environments, such as residential homes and small offices, i.e. indoor environments. Model F represents larger indoor or outdoor propagation environments. Each model has its own parameters as: tap delays, corresponding power, Azimuth Spread (AS), Angle of Arrival/Departure (AOA/AOD). In order to analyze a rich environment and assess the impact of more parameters, the choice was made on the model $\mathrm{D}$ which represents typical offices. The MIMO channel matrix $H$ for each tap, at one instant of time in the power delay profile, models can be separated into a constant (LOS) matrix and a variable Rayleigh matrix.

For a $2 \times 2$ MIMO system, the channel matrix $H[4]$ is

$$
\begin{gathered}
H=\sqrt{P}\left(\sqrt{\frac{K}{K+1}} H_{F}+\sqrt{\frac{1}{K+1}} H_{V}\right)= \\
\sqrt{P}\left(\sqrt{\frac{K}{K+1}}\left[\begin{array}{ll}
e^{j \varphi_{11}} & \frac{e^{j \varphi_{12}}}{\sqrt{10}} \\
\frac{e^{j \varphi_{21}}}{\sqrt{10}} & e^{j \varphi_{22}}
\end{array}\right]+\sqrt{\frac{1}{K+1}}\left[\begin{array}{ll}
X_{11} & \frac{X_{12}}{\sqrt{2}} \\
\frac{X_{21}}{\sqrt{2}} & X_{22}
\end{array}\right]\right)
\end{gathered}
$$

where $X_{\mathrm{NM}}(\mathrm{N}$-th receiving and $\mathrm{M}$-th transmitting antenna) are correlated zero-mean, unit variance, complex Gaussian random variables as coefficients of the variable (Rayleigh) matrix $H_{V}, e^{j \varphi}$ NM are the elements of the constant matrix $H_{F}, K$ is the Rician factor, and $P$ is the power of each tap.

To correlate the $X_{\mathrm{NM}}$ elements of the matrix $X$ [5], the Kronecker product of the transmit and receive correlation matrices is preformed:

$$
\left\{\left[R_{T x}\right] *\left[R_{R x}\right]\right\}^{\frac{1}{2}}\left[H_{i i d}\right]
$$

where $R_{T x}$ and $R_{R x}$ are the transmit and receive correlation matrices respectively, and $H_{i i d}$ is a vector (only here, otherwise it is a matrix) of independent, complex Gaussian random variables with zero mean and unit variance.

$R_{T x}$ and $R_{R x}$ matrices are derived based on the Power Angular Spectrum (PAS) formulation [4-5].This model supposes there is no correlation between transmitting and receiving antennas. The (PAS) for each tap is a function of the antennas spacing, angular spread (AS) and angle of incidence, angle of arrival (AOA) or angle of departure (AOD), depending on $\mathrm{Tx}$ or $\mathrm{Rx}$ of each cluster.

Some angular elements are shown in Fig. 2 for a 3x3MIMO system.

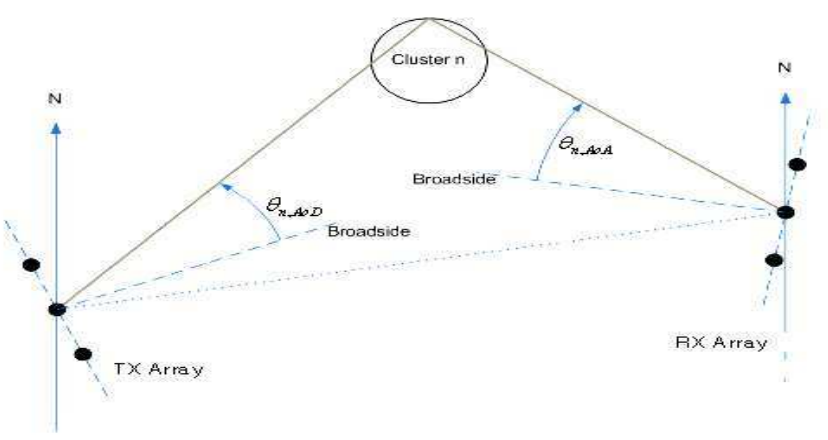

Figure 1 : Angular parameters : AOD/AOA

In Fig. 1, the result of a comparative analysis of four reference channels is released using the simulation environment described above. Curves represent the evolution of packet error rate (PER) versus signal to noise ratio (SNR), for a simple AWGN channel. SNR values moderately low are enough to reach an acceptable PER. Secondly, by comparing both TGN-B channels (indoor residential: two clusters) and TGN-D (large offices: three clusters), we conclude that the 11.n MIMO system takes advantage of the richness of the environment since the model $\mathrm{D}$ is less demanding in terms of SNR than the model B. This can be explained by the proper use of multi-path made by a MIMO system when their delays are below the limit of the guard interval.

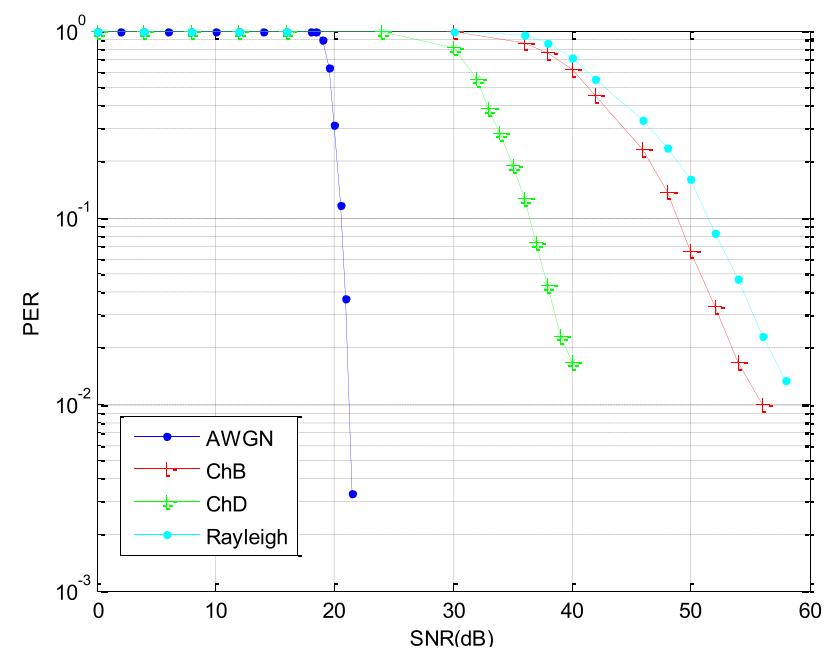

Figure 2: Simulated PER versus SNR for reference wireless channels 


\section{ANGULAR PARAMETERS AND MODULTION AND CODING SCHEME (MCS)}

\section{A. Bit rate adaptation}

The IEEE $802.11 \mathrm{n}$ systems use the 20 or $40 \mathrm{MHz}$ bandwidth within 15 different MCS numbered 1 to 15 , MCS8 to 15 corresponding to the use of 2 spatial streams. Depending on the indoor transmission environment, the system switches from one MCS to another to adapt the bit rate to the link quality imposed by the radio environment: different objects, obstacles between transmitter and receiver, the received power and interference level. The PER is one of the criterion used in order to choose a new MCS because there is a standardized threshold of PER requiring an immediate change of the transmission rate to maintain the link between two devices. For instance, when the propagation channel is degraded, the transition at a lower MCS index, i.e. at a lower bit rate becomes necessary and the throughput is reduced. This value of packet error rate is set by the $802.11 \mathrm{n}$ standard to 0.1 for a packet size of 4096 bytes [6].

Since we use a packet size of 1500 bytes and the studies [7] have shown that the packet error rate varies approximately linearly with the packet size, when the BER is low, the threshold value of the PER that we consider is defined by:

$$
P E R=0.1 \frac{1500}{4096}=0.0366
$$

In the following paragraphs, the effect of three parameters related to the propagation channel is analyzed; two of the transmitter side (angular spread and angle of departure) and the third is related to the receiver (angle of arrival). The aim is to assess the weight of each parameter on a possible degradation of performance in terms of the maximum data rate the system can select to guarantee the PER threshold. To do so, simulations are designed in such a way that we change the value of a parameter for different indices of MCS, particularly for MCS 13, 14 and 15. The evolution of the PER versus the channel parameter is then shown to illustrate in which cases a MCS can be used.

Results given below correspond to an analysis performed on the parameters of the first cluster for model D which, according to our previous studies [1], has the greatest impact when compared to the second or the third cluster.

\section{B. Angular Spread $(A S)$ and $M C S$}

The first observation drawn from Fig. 3 is that the packet error rate decreases when the value of the angular spread increases, i.e. when we have a rich environment between the transmitter and receiver. This propagation environment (with SNR $=38 \mathrm{~dB}$ ), represented in this case by the angular spread, has no effect when using MCS 13 and 14 corresponding to data rates of $104 \mathrm{Mbps}$ and $117 \mathrm{Mbps}$ if $\mathrm{AS}>10^{\circ}$.

MCS 15, which corresponds to a data rate of $130 \mathrm{Mbps}$, can be used for high values of AS, above $27^{\circ}$. This is not always possible because, in indoor, measurements [10] have shown that the angular spread varies between $10^{\circ}$ and $70^{\circ}$. Experimental investigations showed that the global angular spreads ranged between $20^{\circ}$ and $70^{\circ}$, based on the location of the transmitter and receiver in Non Line Of Sight (NLOS) environment. The default value defined by the $\mathrm{D}$ model for the first cluster is $27.7^{\circ}$.

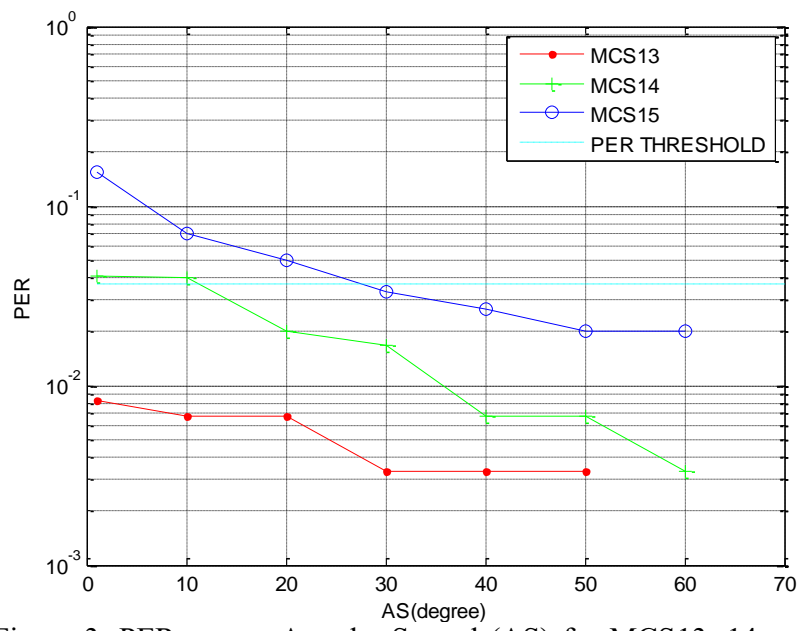

Figure 3: PER versus Angular Spread (AS) for MCS13, 14 and 15. Channel D, SNR $=38 \mathrm{~dB}$

\section{Angle of Departure (AOD) and MCS}

In Fig. 4, the PER is given versus the angle of departure measured for the same MCS as previously. Each curve can be approximately obtained by translating vertically other one. This can be explained by an identical behavior of the system toward the same environment. A maximum PHY bit rate of about $117 \mathrm{Mbps}$ can be achieved for any value of AOD i.e. operating with the MCS14 and 15. For MCS 15 the curve is completely above the PER threshold for changing the modulation and coding scheme.

PER peaks are reached for values of AOD/AOA of about $90^{\circ}$, which corresponds to the fact that a ray parallel to the antenna array array leads to a lower channel correlation that degrades the performance of a link.

The same remarks can be made in the case of the angle of arrival based on Fig. 5 .

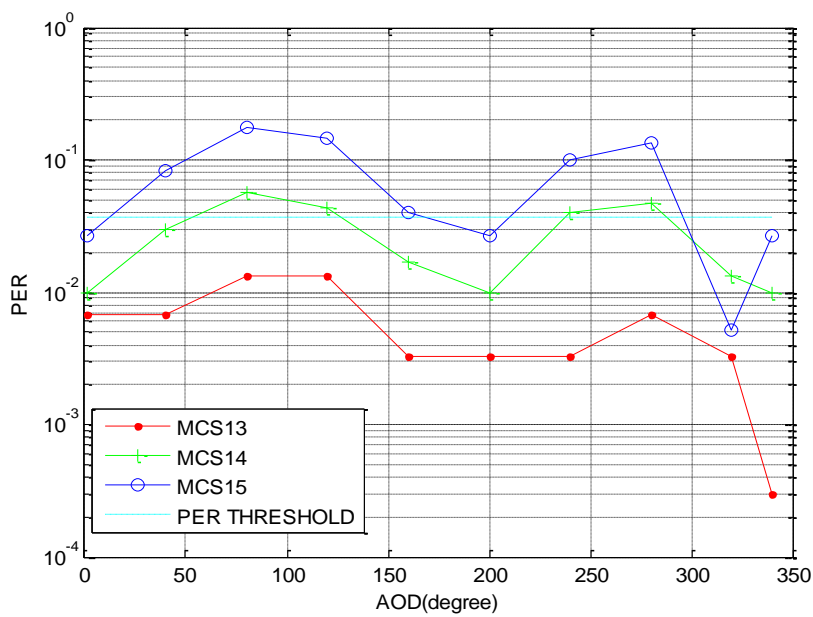

Figure 4: PER versus Angle of Departure (AOD) for MCS13, 14 and 15. Channel D, SNR $=38 \mathrm{~dB}$ 


\section{Angle of Arrival (AOA) and MCS}

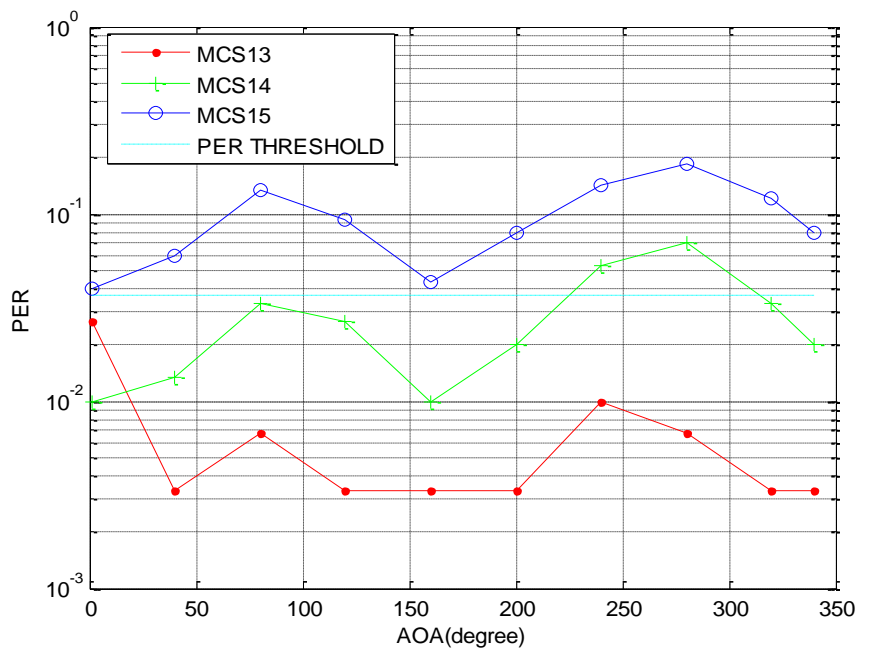

Figure 5: PER versus Angle of Arrival (AOA) for MCS13, 14 and 15. Channel D, SNR $=38 \mathrm{~dB}$

\section{IMPACT OF TWO PARAMETERS}

In this section, the joint impact of two propagation parameters is considered, called $\mathrm{p}_{1}$ and $\mathrm{p}_{2}$. Examples of results give relevant areas, i.e. the pairs $\left(\mathrm{p}_{1}, \mathrm{p}_{2}\right)$ where performance in terms of PER is the best.

In Fig. 6, the evolution of the correlation coefficient of the transmit side $R_{T x}$ versus the angular spread (AS) and the transmitting antenna spacing $\left(\mathrm{d}_{\mathrm{Tx}}\right)$ is presented. $\mathrm{R}_{\mathrm{Tx}}$ depends on the studied parameters and therefore it is able to describe the behavior of the channel and is correlated to the PER [1]. The correlation coefficient remains high even for relatively great values of the antennas spacing when the value of the angular spread is small therefore the $\mathrm{R}_{\mathrm{Tx}}$ is clearly more sensitive to AS than to the distance between antennas. The distances are expressed in terms of wavelength $\lambda$.

In Fig. 7, and as can be expected, the packet error rate follows the same trend as the $\mathrm{R}_{\mathrm{Tx}}$ (in fig.6). It is higher when the spacing between antennas and AS are both low, but a greater spacing between the antennas $\left(\mathrm{d}_{\mathrm{TX}}>\lambda\right)$ can compensate the performance degradation caused by a small value of the angular spread (below $20^{\circ}$ ).

We find the same trends as previously, i.e. the periodicity of PER, even when the study concerns the relationship between the angle of departure and the spacing between transmitting antennas (Fig. 8) or the angular spread (Fig. 9). We show that high values of the distance between transmitting antennas $\left(\mathrm{d}_{\mathrm{Tx}}\right.$ $>\lambda$ ) may be sufficient to reach a relatively acceptable error rate for any value of AOD. In practice, AOA and AOD are random because the access point (AP) and especially the mobile terminal have a random orientation. To ensure the best performance in all configurations, the recommendation is to increase sufficiently the antennas separation (if we have a linear antenna array). The same observation remains valid: values of $\mathrm{AS}$ above $40^{\circ}$ can ensure a PER not too high.

This shows the importance of the spacing of antennas and the angular spread when compared to the angle of departure.

It should be noted that results similar to those presented above were found when studying the receiving side, i.e. the angle of arrival (AOA), angular spread in reception and spacing between receiving antennas.

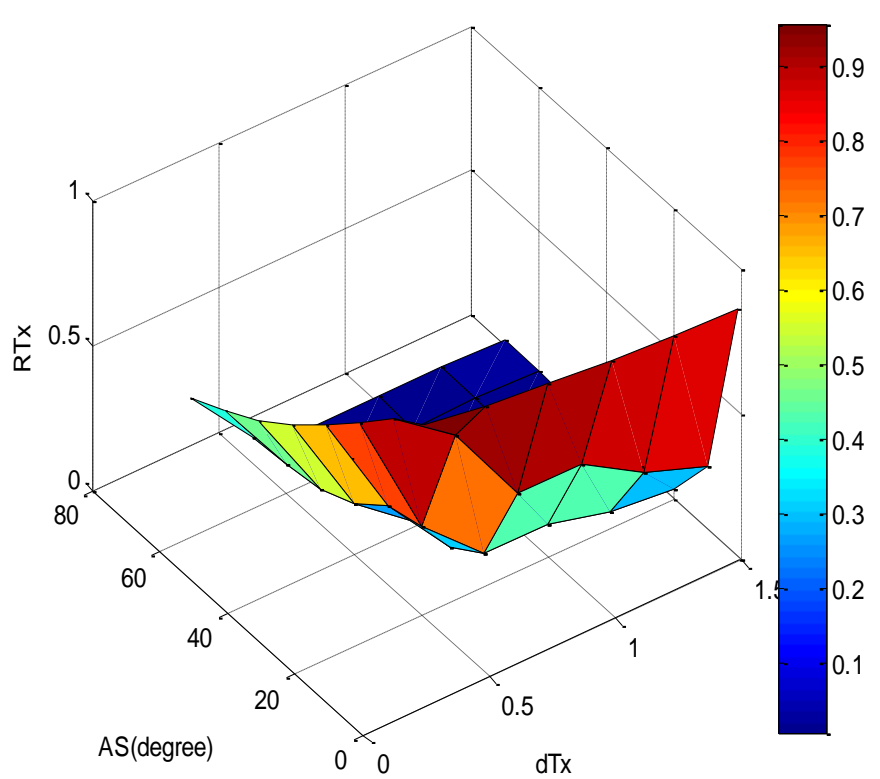

Figure 6: RTx versus Angular Spread and transmitting antennas spacing $(\mathrm{dTx})$

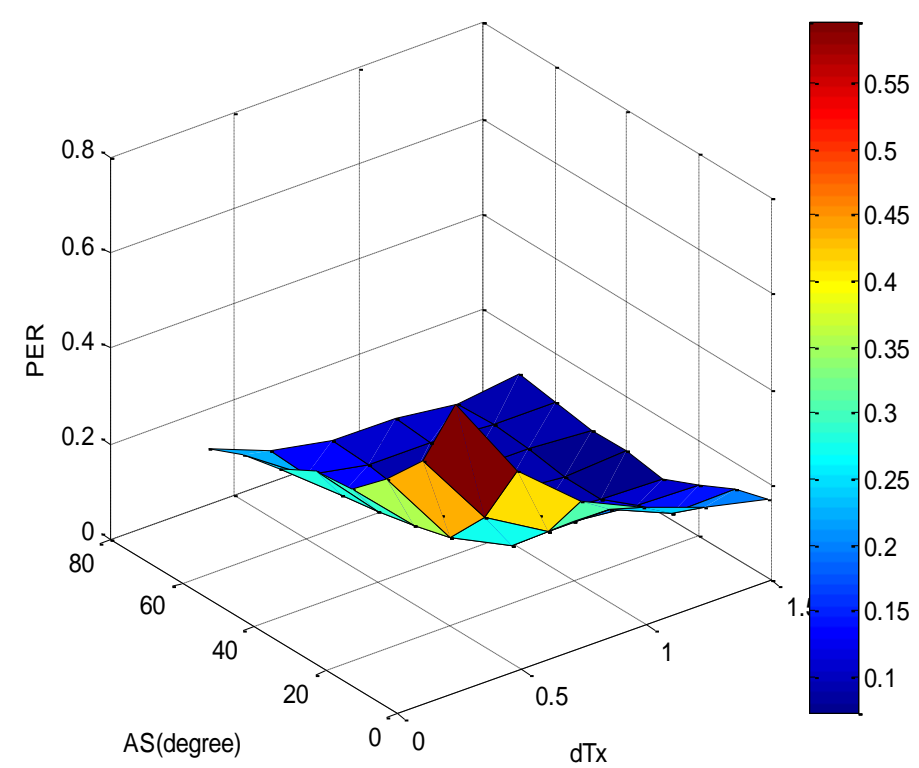

Figure 7: PER versus Angular Spread and transmitting antennas spacing $(\mathrm{dTx})$ 


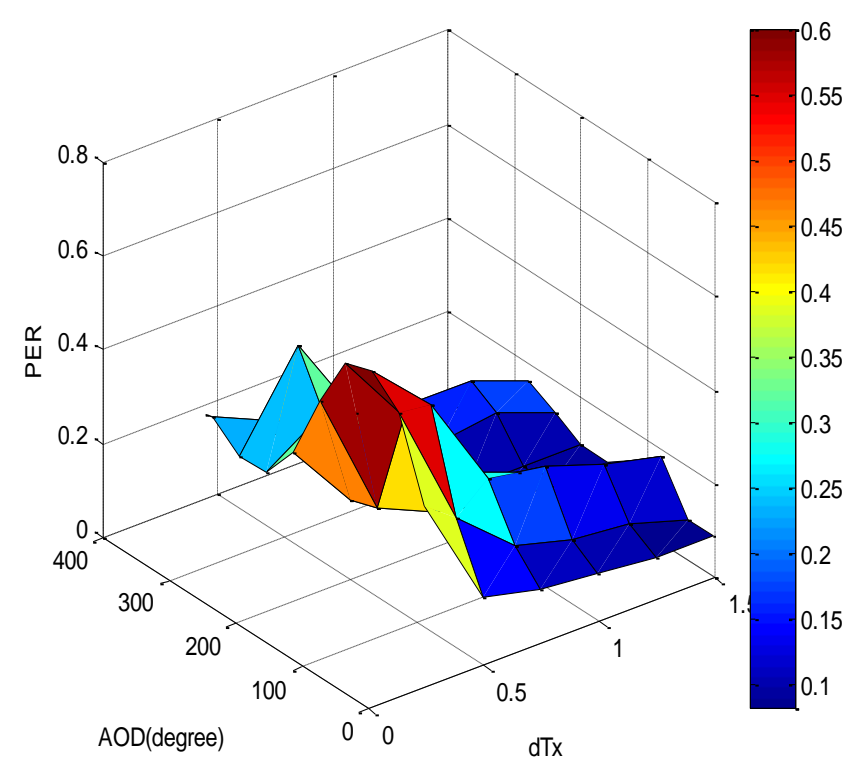

Figure 8: PER versus Angle of Departure and spacing of transmitting antenna (dTx in $\lambda$ )

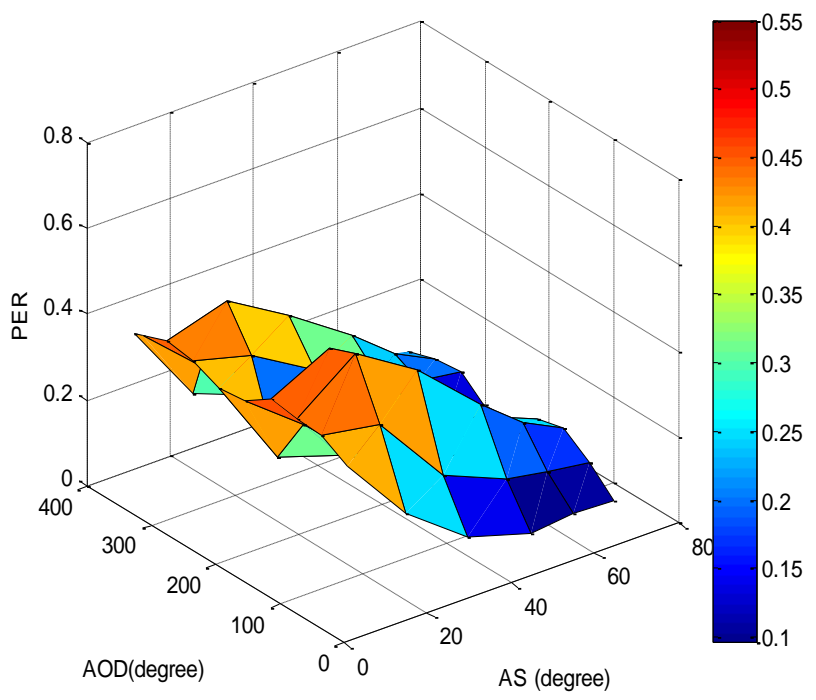

Figure 9: PER versus Angle of arrival and Angular Spread

\section{CONCLUSION}

In this paper, we have presented an explanation of MIMO system performance degradation in the indoor environment, namely the IEEE $802.1 \mathrm{n}$ has been presented. Since the received power is not sufficient to justify the dispersion of throughputs even in a non interfered environment, the impact of some parameters of the wireless channel on PER and on the selected MCS has been studied through several examples. From the obtained results, we can conclude that for a $20 \mathrm{MHz}$ bandwidth with a $2 * 2$ MIMO system and for a same received power it is not always possible to achieve $130 \mathrm{Mbps}$. A lower MCS has to be used, corresponding to a throughout reduction experienced by the user. Secondly, an analysis of the simultaneous effect of two parameters has showed the importance of the distance between antennas and the angular spread compared with the angle of departure to make the performance less sensitive to the channel parameters dispersion.

\section{REFERENCES}

[1] Bouhlel, A.; Guillet, V.; El Zein, G.; Zaharia, G.; , "Impact of wireless propagation channel parameters on IEEE 802.11 n performances," Antennas and Propagation (EUCAP), Proceedings of the 5th European Conference on , pp. 2033-2037, 11-15 April 2011

[2] L. Schumacher and B. Dijkstra, "Description of a Matlab implementation of the indoor MIMO WLAN channel model proposed by the IEEE 802.11 TGn Channel Model Special Committee", Implementation note, version 3.2 - May 2004.

[3] T. K. Paul \& T. Ogunfunmi, "Wireless LAN Comes Of Age: Understanding the IEEE 802.11n Amendment", IEEE Circuits and Systems Magazine, First quarter 2008, vol. 8, pp. 28-54, 2008.

[4] A. Perahia and R. Stacey, "Next Generation Wireless LANs Throughput, Robustness, and Reliability in 802.11n", Cambridge, 2008.

[5] TGn Channel Models, IEEE Std. 802.11--03/940r4, May, 2004.

[6] IEEE Standard for Information technology--Telecommunications and information exchange between systems--Local and metropolitan area networks--Specific requirements Part 11: Wireless LAN Medium Access Control (MAC) and Physical Layer (PHY) Specifications Amendment 5: Enhancements for Higher Throughput.

[7] M. Kiessling and J. Speidel "Analytical performance of MIMO zeroforcing receivers in correlated Rayleigh fading environments", Proc. of the $4^{\text {th }}$ IEEE Workshop on Signal Processing Advances in Wireless Communications, SPAWC 2003, pp. 383-387, 15-18 June 2003

[8] H. Sizun, V. Guillet, and S. Durieux, "Modelisation empirique de la capacité du canal $802.11 \mathrm{ab} / \mathrm{g}$ ", Journées Scientifiques 2009 d'URSI France, 24 et 25 Mars 2009.

[9] A. A. Hutter, F. Platbrood, J. Ayadi, "Analysis of MIMO capacity gains for indoor propagation channels with LOS component", the $13^{\text {th }}$ IEEE International Symposium on Personal, Indoor and Mobile Radio Communications, , vol. 3, pp. 1337- 1341, 15-18 Sept. 2002.

[10] J. Medbo and J. Berg, "Spatio-temporal channel characteristics at 5 $\mathrm{GHz}$ in a typical office environment," in Proc. IEEE Vehicular Technology Conference 2001, VTC-Fall, vol. 3, no. 54, pp. 1256-1260, 7-11 Oct. 2001. 